\title{
Zinc protoporphyrin/haem ratio and plasma ferritin in preterm infants
}

\author{
I J Griffin, M M Reid, K P B McCormick, R J Cooke
}

Arch Dis Child Fetal Neonatal Ed 2002;87:F49-F51

See end of article for authors' affiliations

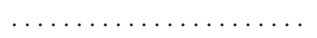

Correspondence to: Dr Griffin, USDA/ARS

Children's Nutrition Research Centre, Baylor College of Medicine, 1100 Bates Street, Houston, TX 77030, USA

igriffin@bcm.tmc.edu

Accepted

8 January 2002

\begin{abstract}
Objective: To study the utility of the zinc protoporphyrin/haem $(\mathrm{ZPP} / \mathrm{H})$ ratio as a measure of iron status in healthy, growing, preterm infants.

Method: ZPP/H was measured in 109 well, preterm infants from the time of hospital discharge until 1 year of age (637 determinations).

Results: ZPP/H was initially high, but steadily declined. This was opposite to what was expected from the known changes in iron stores during the first year of life and the observed changes in plasma ferritin. Subjects with higher ZPP/H ratios tended to have lower ferritins, but changes in ZPP/H in a given subject were poorly reflected by changes in plasma ferritin. Between 6 and 9 months of age, ZPP/H correlated with other measures of iron status, but serum ferritin concentration did not.

Conclusion: Use of the ZPP/H ratio as a measure of iron status during the first year of life appears to be confounded by the developmental changes in $\mathrm{ZPP} / \mathrm{H}$, but in the later half of this period it may be a better measure of iron status than serum ferritin.
\end{abstract}

a ron is an essential nutrient in humans, and deficiency is associated with numerous haematological and nonhaematological manifestations. ${ }^{1}$ Preterm infants have high iron requirements, ${ }^{2}$ and almost invariably develop iron deficiency anaemia in the first year of life without iron supplementation. ${ }^{34}$ This is concerning as iron deficiency anaemia in older infants may lead to developmental delays that do not respond to subsequent iron treatment. ${ }^{56}$

The American Academy of Pediatrics recommends that preterm infants are screened for iron deficiency at 6-9 months of age using packed cell volume or haemoglobin concentration, ${ }^{7}$ but neither of these are particularly sensitive measures of iron status. ${ }^{8}$ Although there is no "perfect" single measure of iron status, ${ }^{9}$ the single most useful measure is serum ferritin. ${ }^{10}$ Although it is widely used, ${ }^{11}$ a cheaper and quicker screening test would be useful.

Zinc protoporphyrin is a metabolic intermediate of the haemoglobin synthetic pathway which accumulates in red blood cells when iron supply is limited. It can be easily measured fluorimetrically and is expressed as a ratio to haem $(\mathrm{ZPP} / \mathrm{H})$. In adults, ZPP/H correlates inversely with plasma ferritin across a wide range of ferritin concentrations, ${ }^{12}$ and is inversely related to the amount of stainable iron in the marrow. ${ }^{13}$ In adults ${ }^{12} 1415$ and children, ${ }^{8} \mathrm{ZPP} / \mathrm{H}$ has been shown to be more sensitive than the packed cell volume or haemoglobin concentration in detecting iron deficiency. It is particularly suited as a screening test because it is cheap, convenient, ${ }^{16}$ and can be carried out on a single drop of blood..$^{10}$

The purpose of this study was to describe the changes in $\mathrm{ZPP} / \mathrm{H}$ in the first year of life in preterm, low birthweight infants, and to test the hypothesis that $\mathrm{ZPP} / \mathrm{H}$ is inversely related to plasma ferritin, as has been shown in adults.

\section{SUBJECTS AND METHODS}

Subjects were part of a prospective study designed to examine the effects of diet on growth and development in preterm, low birthweight infants after hospital discharge. ${ }^{17}{ }^{18}$ Healthy, low birthweight $(<1750 \mathrm{~g})$, preterm $(<34$ weeks gestation) infants were recruited immediately before hospital discharge. Informed consent was obtained from the subject's parents, and the study received local ethical approval.
Infants were seen at discharge, at 38 weeks of postconceptional age, at their expected date of delivery, monthly to 6 months of corrected age, and at 9 months of corrected age. At each visit, blood was taken and preserved with sodium EDTA. Complete blood counts were obtained using a Coulter Counter (model STKS), which was calibrated daily with "4C" calibration solution. Whole blood $\mathrm{ZPP} / \mathrm{H}$ was measured on unwashed red cells using an Aviv ZPHematofluorometer (Lakewood, New Jersey, USA). Plasma ferritin was measured by immunoradiometric assay (Ferritin Mab; ICN Pharmaceuticals, Orangeburg, New York, USA). All assays were carried out in the clinical haematology laboratories of the Royal Victoria Infirmary, Newcastle upon Tyne.

Statistical analysis was carried using the StatView statistical package for Macintosh (version 5.01; SAS Institute, Cary, North Carolina, USA). Simple and multiple regression, analysis of variance, and analysis of covariance were used as appropriate. Results were considered significant at $\mathrm{p}<0.05$. ZPP/H ratios are expressed as $\mu \mathrm{g} / \mathrm{g}$ haemoglobin $(\mathrm{Hb})$ and plasma ferritin as $\mu \mathrm{g} / \mathrm{l}$. ZPP/H and plasma ferritin were log transformed (to the base 10) before analysis to normalise the distribution, as both were positively skewed. Values are quoted as mean (SD), unless stated otherwise.

To study the between subject correlation between $\mathrm{ZPP} / \mathrm{H}$ and plasma ferritin values, $\log (\mathrm{ZPP} / \mathrm{H})$ and $\log$ (ferritin) were regressed against postnatal age and the square of postnatal age (both centred at postnatal age 180 days). Residuals around these regressions were calculated and averaged for each subject. Finally, all the individual average $\log (\mathrm{ZPP} / \mathrm{H})$ residuals were correlated with the corresponding average log (ferritin) residuals. A significant negative correlation would suggest that subjects with higher than average serum ferritin would have lower than average $\mathrm{ZPP} / \mathrm{H}$ ratio (and vice versa).

To study the within subject correlation between $\mathrm{ZPP} / \mathrm{H}$ and plasma ferritin, log $(\mathrm{ZPP} / \mathrm{H})$ and $\log$ (ferritin) were regressed against postnatal age, the square of postnatal age (both centred at postnatal age 180 days). A subject's log (ZPP/H) residuals and log (ferritin) residuals were correlated, and an average correlation for all subjects was calculated. A significant negative correlation would support the hypothesis that changes in ZPP/H in a particular subject would be mirrored by reciprocal changes in plasma ferritin (and vice versa). 


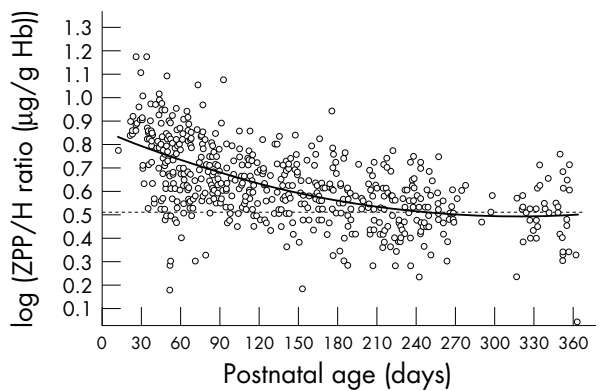

Figure 1 Scattergram of log (zinc protoporphyrin (ZPP/H) $(\mu g / g$ haemoglobin $(\mathrm{Hb})$ ) versus postnatal age. The solid line shows the second order polynomial regression line, and the broken line the upper limit of normal for adults in our laboratory $(3.2 \mu \mathrm{g} / \mathrm{g} \mathrm{Hb})$.

The relation between $\mathrm{ZPP} / \mathrm{H}$ and plasma ferritin at 6-9 months of postnatal age and 6-9 months of corrected age were assessed by simple regression, as was the relation between log $(\mathrm{ZPP} / \mathrm{H})$ or $\log$ (ferritin) and other measures of iron status (haemoglobin concentration, mean cell volume, mean cell haemoglobin, and red cell distribution width). If there was more than one data point for a given subject during either of these time periods, only the first one was used in this part of the analysis.

\section{RESULTS}

A total of 109 infants were recruited, with a mean birth weight of 1345 (271) g, and a gestation of 214 (15) days. Almost half $(48 \%)$ were boys, and most $(60 \%)$ received no blood transfusions before discharge. The remainder received between one and five blood transfusions (median one). No infant had a blood transfusion after hospital discharge.

\section{$\mathrm{ZPP} / \mathrm{H}$ ratios}

Altogether, 637 measurements of $\mathrm{ZPP} / \mathrm{H}$ were made in the first year of life. Up to 11 measurements were made in each subject (range 1-11; median 6). ZPP/H was initially high, fell rapidly, then stabilised (fig 1). Multiple regression analysis shows that $\log (\mathrm{ZPP} / \mathrm{H})$ was related to postnatal age $(t=-3.74, \mathrm{p}=$ $0.0002)$, but not postconceptional age $(t=1.68, \mathrm{p}=0.094)$. Most of the decline in ZPP/H occurred in the first 6 months of postnatal life (fig 1). Analysis of covariance with subject as a covariant showed that $\log (\mathrm{ZPP} / \mathrm{H})$ varied significantly between subjects $(F=4.6, \mathrm{p}<0.001)$ and as a function of postnatal age $(F=138, \mathrm{p}<0.0001)$.

\section{Plasma ferritin}

In the later part of the study, plasma ferritin was measured at the same time as $\mathrm{ZPP} / \mathrm{H}$. A total of 357 samples were taken in 86 subjects. Between 0 and 10 measurements were made in each infant (median four). Plasma ferritin declined with

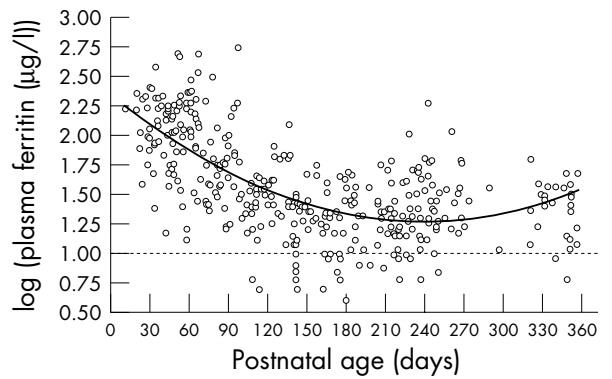

Figure 2 Scattergram of log (plasma ferritin) against postnatal age. The solid line shows the second order polynomial regression line (for log (ferritin)), and the broken line the lower limit of normal for adults in our laboratory.

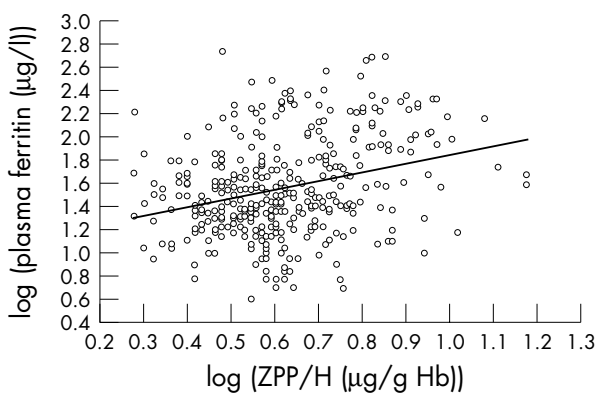

Figure 3 Scattergram of log (plasma ferritin) against log (zinc protoporphyrin $(\mathrm{ZPP} / \mathrm{H})(\mathrm{\mu g} / \mathrm{g}$ haemoglobin $(\mathrm{Hb}))$.

increasing postnatal age (fig 2). It was less than $20 \mu \mathrm{g} / \mathrm{l}$ in 96 instances $(27 \%)$, less than $15 \mu \mathrm{g} / \mathrm{l}$ in 58 instances $(16 \%)$, and less than $10 \mu \mathrm{g} / \mathrm{l}$ in 25 instances (7\%).

\section{Relation between ZPP/H and plasma ferritin during the first year of life}

There was a weak, but significant, correlation between ZPP/H and plasma ferritin $(r=+0.26, \mathrm{p}<0.0001$; fig 3$)$, but it is important to note that the slope of the regression line is positive, not negative (as expected from theoretical considerations).

Residuals of $\log (\mathrm{ZPP} / \mathrm{H})$ and $\log$ (ferritin) were calculated from a multiple regression model that used postnatal age and the square of postnatal age (both centred to a value of postnatal age 180 days). There was a weak $(r=-0.22)$ but significant $(\mathrm{p}=0.038)$ negative correlation between the average log $(\mathrm{ZPP} / \mathrm{H})$ residual for a subject, and the corresponding log (ferritin) residual. This is consistent with the hypothesis that subjects with higher ferritin concentrations have lower ZPP/H ratios (and vice versa).

A similar model that included "subject" was carried out. When a subject's ZPP/H residuals and ferritin residuals (around their own individual line of best fit) were correlated, the average correlation coefficient was -2.91 , but did not differ significantly from zero ( $95 \%$ confidence interval -7.14 to $+1.33 ; \mathrm{p}=0.18)$. In other words, in any subject, changes in $\mathrm{ZPP} / \mathrm{H}$ were poorly mirrored by changes in plasma ferritin.

\section{ZPP/H and plasma ferritin at 6-9 months of age}

$\mathrm{ZPP} / \mathrm{H}$ and plasma ferritin was measured at 6-9 months of postnatal age (175-270 days of postnatal age, 362-477 days of postconceptional age) in 54 subjects. There was a nonsignificant negative correlation between $\log (\mathrm{ZPP} / \mathrm{H})$ and $\log$ (ferritin) $(r=-0.2 \mathrm{l}, \mathrm{p}=0.12)$. Log $(\mathrm{ZPP} / \mathrm{H})$ tended to correlate negatively with haemoglobin concentration $(y=13.0-1.76 x, r=-0.24, p=0.08)$, and correlated significantly with mean cell volume $(\mathrm{y}=88.1-17.2 \mathrm{x}, r=-0.49$, $\mathrm{p}=0.002)$, mean cell haemoglobin $(\mathrm{y}=30.1-5.45 \mathrm{x}$, $r=-0.37, \mathrm{p}=0.006)$, and red cell distribution width $(\mathrm{y}=10.4+5.5 \mathrm{x}, r=+0.43, \mathrm{p}=0.001)$. There was, however, no correlation between log (ferritin) and haemoglobin concentration, mean cell volume, mean cell haemoglobin, or red cell distribution width ( $\mathrm{p}>0.50$ for all comparisons).

$\mathrm{ZPP} / \mathrm{H}$ and ferritin was measured at 6-9 months of corrected age (459-561 days of postconceptional age in 51 subjects). Once again there was no significant correlation between $\log (\mathrm{ZPP} / \mathrm{H})$ and $\log$ (ferritin) $(r=+0.19, \mathrm{p}=0.18)$. However, there was a significant negative correlation of $\log (\mathrm{ZPP} / \mathrm{H})$ with haemoglobin concentration $(r=-0.48, \mathrm{p}=0.004)$, mean cell volume $(r=-0.39, \mathrm{p}=0.005)$, and mean cell haemoglobin $(r=-0.45$, $\mathrm{p}=0.0008)$ and a significant positive correlation with red cell distribution width $(r=+0.39, \mathrm{p}=0.005)$. As before, there was no correlation between log (ferritin) and haemoglobin concentration, mean cell volume, mean cell haemoglobin, or red cell distribution width ( $\mathrm{p}>0.50$ for all comparisons). 


\section{DISCUSSION}

In adults, ZPP/H is inversely correlated with plasma ferritin, ${ }^{12}{ }^{14}$ and inversely related to the amount of stainable iron in the marrow. ${ }^{13}$ We observed a fall in plasma ferritin in the first year of life, which agrees with the known changes in body iron stores in neonates. ${ }^{11}{ }^{19}$ However, rather than seeing the expected increase in $\mathrm{ZPP} / \mathrm{H}$ during this period, a decrease was seen. Indeed $\mathrm{ZPP} / \mathrm{H}$ and ferritin were directly, not inversely, related. When the entire dataset was examined using multiple regression, with age included in the model, there was no relation between $\log (\mathrm{ZPP} / \mathrm{H})$ and $\log$ (ferritin). However, there was an inverse relation between average $\mathrm{ZPP} / \mathrm{H}$ residuals for each subject, from a multiregression model, and corresponding $\log$ (ferritin) residuals. This suggests that subjects with a higher than average plasma ferritin tend to have lower $\mathrm{ZPP} / \mathrm{H}$ (the between subject correlation). However, the relation was relatively weak $(r=-0.22)$. When a similar model was used that incorporated "subject", there was no significant relation between an individuals's ZPP/H residuals (around their own line of best fit) and their ferritin residuals, when the correlation coefficients for all subjects were averaged. In other words, changes in a subject's ZPP/H correlated poorly with changes in their plasma ferritin (the within subject correlation). The positive correlation between $\log (\mathrm{ZPP} / \mathrm{H})$ and $\log$ (ferritin) on simple regression analysis appears to be an artefact of the developmental changes in $\mathrm{ZPP} / \mathrm{H}$, which was high in the early part of the study.

In our study, ZPP/H was measured using a photofluorimetric assay, which can be falsely increased by other fluorophores such as bilirubin. ${ }^{20}$ However, none of our subjects were visibly jaundiced. The effect of other fluorophores can be overcome by using washed red cells, ${ }^{21}{ }^{22}$ but a small study in term infants, using washed red cells, has shown similar $\mathrm{ZPP} / \mathrm{H}$ ratios to our own. ${ }^{21}$

Plasma ferritin is not a perfect measure of iron stores, and may be falsely raised in infective conditions, and there is only indirect evidence of its value as a measure of iron stores in preterm infants. However, low values are felt to be highly suggestive of iron deficiency, and it remains the most widely used measure of iron status. ${ }^{10}{ }^{11}$ None of the subjects in the study were considered to have any infectious condition at the time of blood sampling.

The American Academy of Pediatrics recommends screening for iron deficiency between 6 and 9 months of age. ${ }^{7}$ We could not show a significant inverse relation between $\log (\mathrm{ZPP} / \mathrm{H})$ and $\log$ (ferritin) at either 6-9 months of postnatal age or 6-9 months of corrected age. However, during both time periods, log $(\mathrm{ZPP} / \mathrm{H})$ correlated significantly with other measures of iron status (haemoglobin concentration, mean cell volume, mean cell haemoglobin, and red cell distribution width), whereas no such relation could be shown for log (ferritin). Higher $\mathrm{ZPP} / \mathrm{H}$ ratios (suggestive of poorer iron status) correlated with lower haemoglobin concentration, lower mean cell volume, lower mean cell haemoglobin, and an increased red cell distribution width. The changes in haemoglobin, mean cell volume, mean cell haemoglobin, and red cell distribution width are those expected in iron deficiency. These findings also agree with the results of one small study that has shown a positive correlation between $\mathrm{ZPP} / \mathrm{H}$ and red cell distribution width in preterm infants at the time of hospital discharge. ${ }^{23}$ Although making correlations between different measures of iron status overlooks the different stages of iron deficiency, these results suggest that $\mathrm{ZPP} / \mathrm{H}$ may be a more useful measure of iron status than plasma ferritin concentration in this age range.

It has been suggested that $\mathrm{ZPP} / \mathrm{H}$ may only begin to increase once body iron stores are exhausted and iron delivery to erythroid precursors becomes the rate limiting step in haemoglobin synthesis, ${ }^{922} 24$ although this is disputed..$^{15}$ The former view does not explain the good correlation between $\mathrm{ZPP} / \mathrm{H}$ and plasma ferritin in adults over a wide range of ferritin concentrations. ${ }^{12}{ }^{14}$
We have shown that subjects with higher ZPP/H levels tend to have lower plasma ferritins, although changes in $\mathrm{ZPP} / \mathrm{H}$, for a given subject, did not correlate significantly with changes in plasma ferritin. In the later part of the first year of life, ZPP/H correlated significantly with other measures of iron status, whereas plasma ferritin did not. It is possible therefore that the $\mathrm{ZPP} / \mathrm{H}$ ratio may be a better measure of iron status in this later age group than plasma ferritin concentration. These results underline the difficulty in assessing iron status in preterm infants.

\section{ACKNOWLEDGEMENTS}

We thank E O'Brian Smith for statistical guidance, Michael J R Healy for helpful and patient insights through the peer review process, Marjorie Henderson, Jacqui Smith, Steve Robinson, and Marion Leighton for help with the study, and the nursing and medical staff of the Royal Victoria Infirmary, Newcastle upon Tyne for support and assistance.

\section{Authors' affiliations}

I J Griffin, K P B McCormick, R J Cooke, Neonatal Nutrition Research Group, Royal Victoria Infirmary, University of Newcastle, Newcastle Upon Tyne, UK

M M Reid, Department of Haematology, Royal Victoria Infirmary, University of Newcastle

\section{REFERENCES}

1 Dallman PR, Siimes MA, Stekel A. Iron deficiency in infancy and childhood. Am J Clin Nutr 1980;33:86-118.

2 Dallman PR. Iron. In: Brown ML, ed. Present knowledge in nutrition. 6th ed. Washington: International Life Sciences Institute-Nutritional Foundation, 1990:241-50.

3 James JA, Combes M. Iron deficiency in the premature infant. Significance, and prevention by the intramuscular administration of iron-dextran. Pediatrics 1960;26:368-74.

4 Woodruff CW. Multiple causes of iron deficiency in infants. JAMA 1958;167:715-20.

5 Lozoff B, Jimenez E, Wolf AW. Long term developmental outcome of infants with iron deficiency. N Engl J Med 1991;325:687-94.

6 Walter T. Effects of iron deficiency anaemia on cognitive skills in infancy and childhood. Clin Haematol 1994;7:815-27.

7 American Academy of Pediatrics committee on nutrition. Iron supplementation for infants. Pediatrics 1976;58:765-8.

8 Siegel RM, LaGrone DH. The use of zinc protoporphyrin in screening young children for iron deficiency. Clin Pediatr 1994;33:473-9.

9 Hastka J, Lasserre JJ, Schwarzbeck A, et al. Laboratory tests of iron status: correlation or common sense? Clin Chem 1996;42:718-24.

10 Cavill I, Jacobs A, Worwood M. Diagnostic methods for iron status. Ann Clin Biochem 1986;23:168-71.

11 Ehrenkranz RA. Iron, folic acid and vitamin B12. In: Tsang RC, Lucas A, Uauy R, et al, eds. Nutritional needs of the preterm infant: scientific basis and practical guidelines. Baltimore: Williams and Wilkins, 1993: 177-92.

12 Labbé RF, Finch CA, Smith NJ, et al. Erythrocyte protoporphyrin/heme ratio in the assessment of iron status. Clin Chem 1979;25:87-92.

13 McLaren GD, Carpenter JTN. Erythrocyte protoporphyrin in the detection of iron deficiency. Clin Chem 1975;21:1121-7.

14 Jensen BM, Sandø SH, Grandjean P, et al. Screening with zinc protoporphyrin for iron deficiency in non-anaemic female blood donors. Clin Chem 1990;36:846-8.

15 Labbé RF, Rettmer RL, Shah AG, et al. Zinc protoporphyrin, past, present, and future. Ann N Y Acad Sci 1987;514:7-14.

16 Schifman RB. Red cell protoporphyrin: an alternative to serum ferritin for assessing iron depletion [Abstract]. Clin Chem 1982;28:1628.

17 Cooke RJ, Griffin IJ, McCormick K, et al. Feeding preterm infants after hospital discharge: effect of dietary manipulation on nutrient intake and growth. Pediatr Res 1998;43:355-60.

18 Griffin IJ, Cooke R, Reid MM, et al. Iron nutritional status in preterm infants fed formulas fortified with iron. Arch Dis Child Fetal Neonatal Ed 1999:81:F45-9.

19 Seip M, Halvorsen S. Erythrocyte production and iron stores in premature infants during the first months of life. Acta Paediatr 1956;45:600-17.

20 Karacic V, PrpicMajic D, Telisman S. The relationship between zinc protoporphyrin (ZPP) and 'free' erythrocyte protoporphyrin (FEP) in lead-exposed individuals. Int Arch Occup Environ Health 1980:47: 165-77.

21 Bartels PC, Helleman PW, Soons JBJ. Interference of plasma fluorophores on the red blood cell zinc protoporphyrin:haemoglobin ratio as determined on a haematofluorimeter. Ann Clin Biochem 1989;26:368-73.

22 Garrett S, Worwood M. Zinc protoporphyrin and iron deficient erythropoiesis. Acta Haematol 1994;91:21-5.

23 Winzerling JJ, Kling PJ. Iron-deficient erythropoiesis in premature infants measured by blood zinc protoporphyrin/heme. J Pediatr 2001;139:134-6.

24 Hastka J, Lasserre JJ, Schwarzbeck A, et al. Central role of zinc protoporphyrin in staging iron deficiency. Clin Chem 1994;40:768-73. 